\title{
In-vitro development of preimplantation embryos recovered from IUD-bearing mice
}

\author{
P. R. Hurst, Kathryn Jefferies, Karin J. Dawson and P. Eckstein \\ Department of Anatomy, Medical School, University of Birmingham, \\ Birmingham B15 2TJ, U.K.
}

\begin{abstract}
Summary. Embryos were collected from the oviducts and uterine horns of mice bearing a unilateral IUD and of non-IUD control mice at various times p.c., examined for the presence or absence of polymorphonuclear leukocytes (PMNLs) and cultured. Normal numbers of embryos were obtained on the IUD side at $48 \mathrm{~h}$ p.c., but reduced numbers were recovered at 60-66 and $72 \mathrm{~h}$ p.c. The numbers of embryos that hatched from those collected from control, IUD-bearing and contralateral non-IUD bearing tracts were, respectively, 81,29 and $36 \%$ for 48 -h embryos, 78,47 and $51 \%$ for 60-66-h embryos, and 88,47 and $54 \%$ for $72-\mathrm{h}$ embryos. At $48 \mathrm{~h}$ and $60-66 \mathrm{~h}$ p.c. the arrest of development occurred at the expanded blastocyst stage, but at $72 \mathrm{~h}$ p.c. some compacted morulae were affected. Some embryos associated with PMNLs at collection developed normally when the PMNLs were dispersed before culture. An initial association with leukocytes is therefore not necessarily detrimental to the subsequent in-vitro development of the embryo in mice.
\end{abstract}

\section{Introduction}

In mice fitted with a unilateral silk thread preimplantation embryos in the uterus were degenerated and surrounded by predominantly polymorphonuclear leukocytes (PMNLs) (Hurst, Jefferies, Eckstein \& Wheeler, 1977a). Histological sections of the uteri revealed the presence of leukocytes within some of the embryos. This close PMNL-embryo association was observed in horns with a silk-thread IUD and the untreated contralateral horn within $12 \mathrm{~h}$ after the time (about $60 \mathrm{~h} \mathrm{p.c.)}$ embryos normally enter the uterus in the LACA strain of mouse, but was not seen in control horns. These observations indicated that PMNLs may be actively involved in the prevention of implantation in mice bearing an IUD. In other species, however, PMNLs are less evident, and it has been suggested that the acellular luminal contents might be embryotoxic (Parr, Schaedler \& Hirsch, 1967; De Boer \& Anderson, 1971; Smith, El Sahwi, Wilson \& Moyer, 1971; see also Moyer, Shaw \& Fu, 1976), thereby rendering embryos in some way abnormal before coming into contact with PMNLs.

It therefore seemed worthwhile to examine early embryos recovered from the oviducts and uterine horns of IUD-bearing mice up to $72 \mathrm{~h}$ p.c., and to investigate the ability of such embryos, whether initially associated with PMNLs or not, to continue further development in vitro. In addition, attempts were made to confirm the previous finding (Hurst et al., 1977a) that embryos collected from an IUD-containing uterine horn are actively invaded by leukocytes.

\section{Materials and Methods}

Virgin LACA mice were used for all experiments. Silk suture (6-0 gauge) was used as the IUD material which was inserted into the middle third of randomly selected single horns at laparotomy. The contralateral horns were left untouched and unoperated animals served as controls. The animals were rested for 3 weeks before being mated during the final hour $(09: 00-10: 00 \mathrm{~h})$ of the 14-h dark period. The mice were killed at 48, 60-66 and $72 \mathrm{~h}$ post coitum (p.c.). Embryos were recovered by flushing either the oviducts or uteri with medium and after being pooled were placed, usually as 
groups of 5 , in drops of medium under equilibrated paraffin oil (Boots, Nottingham) in a $5 \%$ $\mathrm{CO}_{2}: 95 \%$ air atmosphere at $37^{\circ} \mathrm{C}$. The medium was the modified Brinster's (1965) medium described by Breed, Peplow, Jefferies \& Eckstein (1973). The initial stage of development of each embryo was recorded and the cultures were examined daily to monitor their subsequent growth. Culture of embryos collected at $48 \mathrm{~h}$ p.c. was maintained for 7 days, but embryos collected at later stages were cultured for 5 days only because by then they had either hatched or degenerated.

Embryos which were grossly degenerate when collected were discarded. Embryos with adherent leukocytes which could not be displaced by repeated manipulation with micropipettes were fixed in $2.5 \%$ glutaraldehyde, processed through an alcohol series and embedded in Spurr or TAAB resin. Semi-thin (about $1 \mu \mathrm{m}$ ) sections were cut on a Porter-Blum MT-2 ultramicrotome and stained with $0.5 \%$ toluidine blue in $0.5 \%$ borax before being examined by light microscopy.

\section{Results}

\section{Cultures}

The state of embryos at collection and after culture is summarized in Tables 1 and 2.

48 h p.c. All the embryos collected from the oviducts of control mice developed from the 4-cell to the compacted morula stage. Thereafter, some became degenerate on each day of culture but the final hatching rate was high.

The embryos from both oviducts of the IUD-bearing mice appeared healthy and developed normally into expanded blastocysts. Thereafter, blastocysts from the IUD-bearing horns showed a significant $(P<0.001)$ arrest in development, with fewer embryos hatching from their zonae.

Of the embryos from the contralateral horns, proportionately fewer expanded blastocysts started hatching, but the values were not significantly different from those of controls except when comparison was made for the completion of hatching $(P<0.001)$.

No PMNLs were seen associated with any of the embryos obtained at $48 \mathrm{~h} p . c$.

60-66 h p.c. All but one (a 4-cell egg) of the embryos from the control mice were morulae and the hatching rate was high (Table 2).

No embryos were recovered from the ipsilateral oviducts or horns of 4 of the 13 mice with a unilateral IUD. In the remaining 9 animals, 7 morulae and two 2 -cell embryos were flushed from a single oviduct. Seven morulae from 3 uterine horns were surrounded by PMNLs, but the latter became dissociated from them shortly after being placed in culture medium, and each of these embryos hatched normally. The remaining 14 morulae had no associated PMNLs. As at 48 h p.c., the numbers of expanded blastocysts hatching were reduced and a correspondingly greater number of embryos was considered to be degenerating by Day 5 in vitro (Table 1).

Embryos were recovered from 12 uterine horns and 1 oviduct on the contralateral side of IUDbearing mice. Nine embryos from 2 uteri were surrounded by PMNLs, which became dissociated after collection: only 5 embryos hatched completely, the others degenerating at the small blastocyst stage by Day 2 in vitro. For the embryos not associated with PMNLs, the pattern of degeneration and developmental arrest was similar to that of embryos from the IUD-bearing horns (Tables 1 and 2).

72 h p.c. The embryos from the control mice were at the morula to expanded blastocyst stage at collection, and high proportions hatched (Table 2).

No embryos were obtained from the IUD side of 4 of the 18 experimental mice. Some were recovered from the oviducts of 4 animals and 6 of the 14 embryos hatched, but the other 8 degenerated within 1 or 2 days in culture. Of the embryos recovered from the uterus, 3 were initially degenerated although not associated with PMNLs, 5 were surrounded by PMNLs, which were dispersed, and 3 hatched. The rate of degeneration in vitro of the other embryos in this group was rapid, almost half having degenerated by Day 2 of culture and failing to develop beyond the compacted morula stage.

Most of the embryos from the contralateral side of mice with a unilateral IUD were obtained from the uterine horns. All 13 embryos recovered from oviducts degenerated in culture before hatching had started. Some of the uterine embryos were degenerate when collected and 4 of the 8 


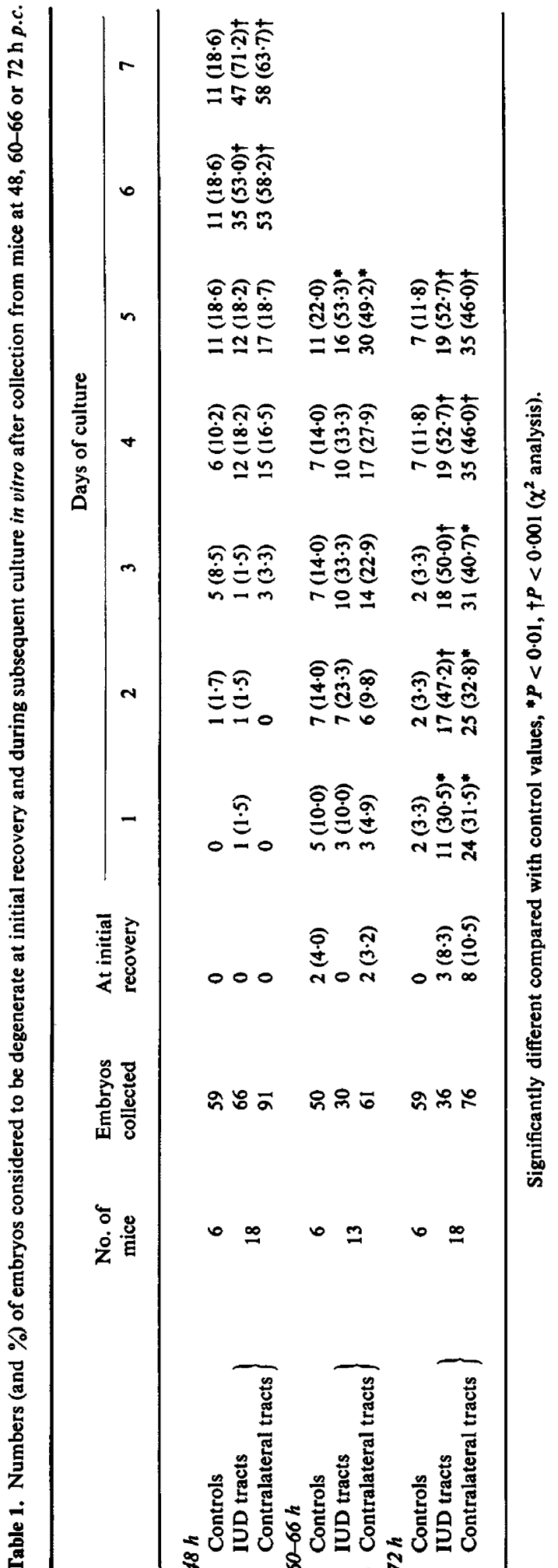


had probably been invaded by PMNLs. The pattern of degeneration and development was again similar to that of embryos from the IUD-containing horns: progression from the compacted morula stage was arrested and significantly more embryos had degenerated by Day 1 of culture (Tables 1 and 2).

Table 2. Numbers (and $\%$ ) of embryos reaching various developmental stages in vitro after recovery from mice at $48,60-66$ or 72 h p.c.

\begin{tabular}{|c|c|c|c|c|c|c|}
\hline & \multicolumn{2}{|c|}{ Morulae } & \multicolumn{4}{|c|}{ Blastocysts } \\
\hline & Early & Compacted & Small & Expanded & Hatching & Hatched \\
\hline \multicolumn{7}{|l|}{$48 h$} \\
\hline Controls & $59(100)$ & $59(100)$ & $58(98 \cdot 3)$ & $54(91 \cdot 5)$ & $53(89 \cdot 8)$ & $48(81 \cdot 4)$ \\
\hline IUD tracts & $66(100)$ & $64(96.9)$ & $61(92 \cdot 4)$ & $58(87.8)$ & $41(62 \cdot 1) \ddagger$ & $19(28.8) \ddagger$ \\
\hline Contralateral tracts & $91(100)$ & $91(100)$ & $82(90 \cdot 1)$ & $77(84 \cdot 6)$ & $70(76 \cdot 9)$ & $33(36 \cdot 3) \dagger$ \\
\hline \multicolumn{7}{|l|}{$60-66 \mathrm{~h}$} \\
\hline Controls & $48(96 \cdot 0)$ & $45(90 \cdot 0)$ & $45(90 \cdot 0)$ & $44(88 \cdot 0)$ & $42(84 \cdot 0)$ & $39(78 \cdot 0)$ \\
\hline IUD tracts & $28(86.6)$ & $28(86 \cdot 6)$ & $24(80.0)$ & $24(80.0)$ & $16(53.3)^{*}$ & $14(46 \cdot 6)^{*}$ \\
\hline Contralateral tracts & $59(96 \cdot 7)$ & $59(96 \cdot 7)$ & $58(95 \cdot 1)$ & $53(86.9)$ & $37(60 \cdot 6)^{*}$ & $31(50.8) \dagger$ \\
\hline \multicolumn{7}{|l|}{$72 h$} \\
\hline Controls & $59(100)$ & $59(100)$ & $59(100)$ & $56(94 \cdot 4)$ & $53(89 \cdot 8)$ & $52(88 \cdot 2)$ \\
\hline IUD tracts & $33(91 \cdot 6)$ & $33(91 \cdot 6)$ & $22(61 \cdot 1) \ddagger$ & $19(52 \cdot 7) \ddagger$ & $17(47 \cdot 2) \ddagger$ & $17(47 \cdot 2)$ \\
\hline Contralateral tracts & $65(85 \cdot 6)$ & $65(85 \cdot 6)$ & $51(67 \cdot 1) \ddagger$ & $50(65 \cdot 8) \ddagger$ & $41(53 \cdot 8) \ddagger$ & $41(53.8) \ddagger$ \\
\hline
\end{tabular}

Significantly different compared with control values; ${ }^{*} P<0.05, \dagger P<0.01, \ddagger P<0.001$ ( $\chi^{2}$ analysis).

\section{Morphology}

Histological sections of embryos collected at $72 \mathrm{~h}$ p.c. from the uteri of mice with an IUD revealed the presence of PMNLs among the blastomeres of zona-intact embryos. A compacted morula was detected within a particularly dense mass of PMNLs (PI. 1, Fig. 1). This embryo was, however, not penetrated, and at this level of resolution did not appear to be abnormal (Pl, 1, Fig. 2). In other morulae into which PMNLs had penetrated (Pl. 1, Figs 3 and 4), areas of cell separation and fragmentation were present. For comparison, a very early blastocyst recovered from the uterine horn of a normal mouse at $74 \mathrm{~h}$ p.c. is shown in Pl. 1, Fig. 5.

\section{Discussion}

With the culture conditions employed during this investigation, from 78 to $88 \%$ of all embryos obtained between 48 and $72 \mathrm{~h}$ p.c. from control mice developed to hatched blastocysts. Further growth stages (e.g. trophoblastic outgrowth; Sherman, 1975) were not examined since the main object of the present study was to assess the potential of embryos for preimplantation development following initial exposure to an IUD environment in vivo.

At $48 \mathrm{~h}$ p.c. a satisfactory yield of tubal embryos was obtained from both sides of the tract of the mice with a unilateral IUD and the growth of the embryos in vitro was comparable to that of controls for 5 days, but then a block to hatching appeared, with embryos becoming arrested at the expanded blastocyst stage. The reason for this arrest is not clear but could be related to a deficiency of some factor essential for hatching or to the presence of an embryotoxic agent. A similar arrest was obtained with embryos collected from the oviduct or uterus at $60-66 \mathrm{~h} p . c$., but at this time and at $72 \mathrm{~h} \mathrm{p.c.} \mathrm{there} \mathrm{was} \mathrm{a} \mathrm{relatively} \mathrm{low} \mathrm{yield} \mathrm{of} \mathrm{embryos} \mathrm{from} \mathrm{the} \mathrm{IUD} \mathrm{sides} \mathrm{of} \mathrm{the} \mathrm{tracts,}$ e.g. 2 embryos/horn compared with 4.9/horn in control mice. This reduced yield has been reported before for mice (Doyle \& Margolis, 1966; Marston \& Kelly, 1969), but the rapid investment of embryos by the large mass of PMNLs seen in histological sections (Hurst et al., 1977a) makes their recovery and identification after flushing difficult. The present observations suggest that contact between PMNLs and embryos, shortly after the latter's entry into the uterus, is not necessarily. 

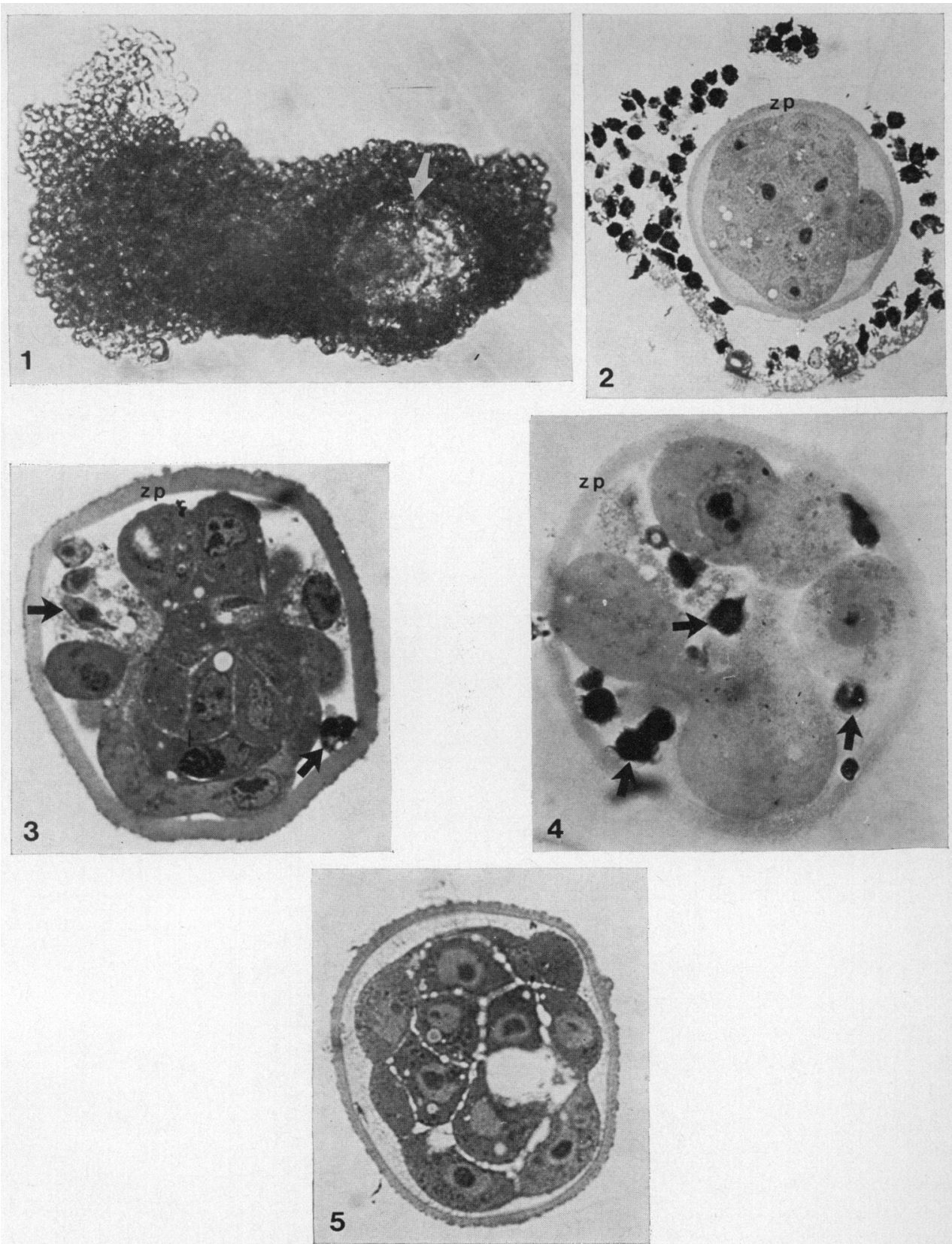

Fig. 1. Large cluster of PMNLs surrounding embryo (arrow) recovered at $72 \mathrm{~h}$ p.c. from a horn containing an IUD. $\times 190$.

Fig. 2. Section through the embryo shown in Fig. 1. Note the absence of penetrated PMNLs and the normal appearance of a compacted morula. $\mathrm{zp}=$ zona pellucida. $\times 400$.

Fig. 3. Section through a compacted morula at $72 \mathrm{~h}$ p.c. from a horn containing an IUD. The arrows indicate penetrated PMNLs. Note discrete areas of cell fragmentation and separation. $z p=$ zona pellucida. $\times 500$.

Fig. 4. Section through a degenerated morula collected from an IUD-bearing horn at $72 \mathrm{~h} p . c$. showing numerous PMNLs (arrows) within the embryo. $z p=$ zona pellucida. $\times 700$.

Fig. 5. Early blastocyst recovered at $74 \mathrm{~h}$ p.c. from the uterus of a (non-IUD) control mouse. $\times 500$. 
deleterious because embryos collected at $60-66 \mathrm{~h}$ p.c. went on to hatch in culture after dispersal of the PMNLs.

At $72 \mathrm{~h}$ p.c. (when embryos have spent about $12 \mathrm{~h}$ in utero), a marked arrest in growth of about $30 \%$ of embryos from IUD-bearing horns was seen after only 1 day in vitro irrespective of any association with PMNLs. Other embryos, however, although initially surrounded by PMNLs went on to hatch after the latter had been dispersed.

Very few embryos, obtained from normal rats, could be recovered $2 \frac{1}{2}-4 \mathrm{~h}$ after being transferred into the horns of rats with an IUD, and those that could be collected showed a greatly increased mortality when subsequently re-transplanted to normal uteri (De Boer \& Anderson, 1971). The experiment of De Boer \& Anderson (1971) demonstrated the marked embryotoxic environment of an IUD uterus but did not specifically investigate the role leukocytes may play, for example, in preventing the recovery of most embryos. In other studies with rats, Fuentealba \& Croxatto (1970) demonstrated a possible 'lytic' effect of the IUD on 5-day embryos, in so far as there was a reasonable recovery of embryos only when a ligature was placed between the IUD and the uterotubal junction; the possible involvement of leukocytes was not analysed. The introduction of live neutrophils, collected from a tract bearing an IUD, into Day-4 pregnant rats significantly reduced implantation (Bo, Krueger \& Sain, 1976), but the nature of the effect, either direct or through toxic products secreted or released by the neutrophils while dying, was not determined. Nevertheless, these experiments support the view that PMNLs could play a major role in the prevention of implantation. Findings consistent with this conclusion have also been obtained in rhesus monkeys (Hurst, Jefferies, Eckstein, Dawson \& Wheeler, 1977b).

From our present and previous results (Hurst et al., 1977a) it would appear that, in mice bearing an IUD, the developing embryo encounters either (i) a hostile non-cellular environment in the oviduct and/or uterus, or (ii) leukocytes in utero which attach to and subsequently penetrate embryos. These two mechanisms may operate, separately or jointly, in the mouse to suppress implantation in both uterine horns of animals bearing a unilateral IUD (Doyle \& Margolis, 1966).

We thank Mrs Janet Parker for her help with the illustrations and Mrs Gladys Macbeth for secretarial assistance. The support of the Ford Foundation (Grant no. 639-0576B) is gratefully acknowledged.

\section{References}

Bo, W.J., Krueger, W.A. \& SAIN, L.E. (1976) Effect of direct injection of neutrophils from uterine horns of rats containing intrauterine devices into recipient pregnant uteri. Fert. Steril. 27, 1318-1321.

Breed, W.G., Peplow, P.V., Jefferies, C. \& Eckstein, P. (1973) Effect of uterine fluid from mice with IUDs, and various chemical compounds, on mouse eggs in culture. Contraception 8, 343-356.

BRINSTER, R.L. (1965) Studies on the development of mouse embryos in vitro. IV. Interaction of energy sources. J. Reprod. Fert. 10, 227-240.

DE BOER, K.F. \& ANDERSON, L.L. (1971) Survival of embryos transferred into the IUD-bearing uterus of the rat. J. Reprod. Fert. 25, 375-386.

DoYLE, L.L. \& MARGolis, A.J. (1966) The effect of an IUFB on reproduction in mice. $J$. Reprod. Fert. 11, 27-32.

Fuentealba, B. \& Croxatto, H.B. (1970) Role of intrauterine environment in the disappearance of eggs in rats bearing an IUD. Contraception 2, 179-185.

Hurst, P.R., Jefferies, K., Eckstein, P. \& Wheeler, A.G. (1977a) Intrauterine degeneration of embryos in IUD-bearing mice. $J$. Reprod. Fert. 50, 187-189.
Hurst, P.R., Jefferies, K., Eckstein, P., Dawson, K. \& Wheeler, A.G. (1977b) Leukocytes are consistently associated with degenerating embryos in IUD-bearing rhesus monkeys. Nature, Lond. 269, 331-333.

Marston, J.H. \& Kelly, W.A. (1969) The time and site of contraceptive action of an intra-uterine device in the mouse. $J$. Endocr. 43, 83-93.

MOYeR, D.L., SHAw, S.T. \& FU, J.C. (1976) Mechanism of action of intrauterine contraceptive devices. In Regulation of Human Fertility, pp. 174-192. Eds K. S. Moghissi \& T. N. Evans. Wayne State University Press, Detroit.

Parr, E.L., Schaedler, R.W. \& Hirsch, J.G. (1967) The relationship of polymorphonuclear leukocytes to infertility in uteri containing foreign bodies. J. exp. Med. 126, 523-538.

SHERMAN, M.I. (1975) Long term culture of cells derived from mouse blastocysts. Differentiation 3, 51-67.

Smith, D.M., El Sahwi, S., Wilson, N. \& MoYer, D.L. (1971) Effect of polymorphonuclear leukocytes on the development of mouse embryos cultured from the two cell stage to blastocysts. Biol. Reprod. 4, 74-83. 